\title{
Interprofessional medication assessment among home care patients: any impact on functioning? Results from a randomised controlled trial
}

\author{
K. Auvinen ${ }^{1,2^{*}}\left(\mathbb{D}, A\right.$. Voutilainen ${ }^{2} \mathbb{D}$, J. Jyrkkä $^{3}$, E. Lönnroos ${ }^{2}$ and P. Mäntyselkä ${ }^{2,4}$ (D)
}

\begin{abstract}
Background: Multimorbidity and polypharmacy are related to the use of potentially inappropriate medicines and negative clinical outcomes including drug-related adverse events and functional declines. Home care clients are a vulnerable patient group often exposed to these risks. The aim of this study was to examine whether an interprofessional medication assessment can influence the functioning of home care patients.

Methods: The FIMA study was a randomised controlled intervention study comparing a general practitioner-led interprofessional medication assessment conducted at the baseline of the study with usual care with a six-month follow-up. We used linear mixed models (LMM) with a random subject effect to detect differences between the usual care and intervention groups in the following outcome measures; Katz index of Activities of Daily Living (ADL), Lawton and Brody scale of Instrumental Activities of Daily Living, Timed up and go-test (TUG), Mini-Mental State Examination, Geriatric Depression Scale and the 3-level version of EQ-5D.

Results: Home care patients $(n=512)$ had major disease burdens and functional limitations. Regarding TUG times, the LMM detected a one second improvement in the FIMA group and $2.4 \mathrm{~s}$ worsening in the usual care group. However, the result was not statistically significant. The ADL revealed an interaction across time, treatment and sex $(p=0.026)$. The ADL score decreased in both groups; the decline being the steepest among women in the intervention group.

Conclusions: In general, medication assessments may have limited impact on functioning of older people. Nonetheless, the FIMA intervention may prevent worsening of mobility among older home care patients.

Trial registration: The Interprofessional Medication Assessment for Older Patients, Clinical Trials.gov. NCT02398812. First registration, 26 March 2015. Retrospectively registered.
\end{abstract}

Keywords: Functioning, Home care, Medication therapy management, Medicines, Older people

\footnotetext{
* Correspondence: kati.auvinen@sosteri.fi

${ }^{1}$ The East Savo Hospital District, BOX 111, Fl-57101 Savonlinna, Finland

${ }^{2}$ Institute of Public Health and Clinical Nutrition, Faculty of Health Sciences, University of Eastern Finland, Kuopio, Finland

Full list of author information is available at the end of the article
}

(c) The Author(s). 2020 Open Access This article is licensed under a Creative Commons Attribution 4.0 International License, which permits use, sharing, adaptation, distribution and reproduction in any medium or format, as long as you give appropriate credit to the original author(s) and the source, provide a link to the Creative Commons licence, and indicate if changes were made. The images or other third party material in this article are included in the article's Creative Commons licence, unless indicated otherwise in a credit line to the material. If material is not included in the article's Creative Commons licence and your intended use is not permitted by statutory regulation or exceeds the permitted use, you will need to obtain permission directly from the copyright holder. To view a copy of this licence, visit http://creativecommons.org/licenses/by/4.0/. The Creative Commons Public Domain Dedication waiver (http://creativecommons.org/publicdomain/zero/1.0/) applies to the data made available in this article, unless otherwise stated in a credit line to the data. 


\section{Background}

The number of home care patients is increasing rapidly with the ageing of the population. A key requirement for living at home in old age is maintaining physical and psychosocial functioning. Functional declines have been clearly associated with medication-related problems in vulnerable older people $[1,2]$. Potentially inappropriate prescribing and drug-related adverse events increase the risks of cognitive impairment, falls and hospital admissions [3, 4].

Several studies have evaluated the impacts of medication assessments in reducing the complexity of medications and inappropriate prescribing [5-7], but less attention has been given to concrete health outcomes such as drug-related adverse events, functioning, general health and quality of life $[8,9]$. The scarcity of studies concerning these health outcomes might partly be because it is very challenging to design a practical and feasible medication assessment model for older patients with multimorbidities and being administered polypharmacy.

An interprofessional team method has been suggested as a solution to promote the rational medicine use among older people [10]. The Finnish Interprofessional Medication Assessment (FIMA) is a phyician led, repeatable and pragmatic model for medication optimization of older people [11]. The FIMA model was developed for home care settings. Baseline findings of the FIMA study showed that home care patients had a significant disease burden. The majority of patients (87\%) had excessive polypharmacy ( $\geq 10$ medicines), clinically relevant drug-druginteractions (74\%) and a risk of drug-induced impairment in renal function (85\%). Functional limitations including mobility and balance problems were also common in this patient group. In the present study, we examined whether the FIMA intervention exerted any effects on the physical, cognitive, and psychosocial functioning, or the healthrelated quality of life of home care patients.

\section{Methods}

\section{Study design and participants}

The FIMA study was a randomized, controlled intervention study involving a comparison between a physician led interprofessional medication assessment and usual care in public home care settings. The complete study design of the FIMA study has been published previously [11]. The Research Ethics Committee of Northern Savo Hospital District and Kuopio University Hospital approved the FIMA study protocol on February 3, 2015. The FIMA study was registered with Clinical Trials.gov on March 20, 2015 (identifier: NCT02398812). Reporting follows the CONSORT 2010 statement.

We screened and recruited patients receiving regular home care services in the study areas. The inclusion criteria were age $\geq 65$ years and registration to public home care services, and at least one of the following: $\geq 6$ medicines in use, dizziness, orthostatic hypotension or a recent fall. We excluded patients whose medication was not managed by the home care and patients undergoing active cancer therapy.

A total of 512 patients were recruited (Fig. 1). The characteristics of the participants have been described previously [11]. Written informed consent was obtained from all individual patients included in the study or their closest proxy if the patient had cognitive impairments. After baseline measurements, patients were randomized to receive the intervention or care-as-usual. Intervention and usual care groups were treated similarly except for the interprofessional medication assessment.

\section{Data collection}

Medication use and patients' diagnoses were verified according to a structured protocol [11, 12]. Comorbidity was defined according to a modified Charlson Comorbidity Index (CCI) [13]. Performance in daily activities, the patient's physical and cognitive performance, depressive symptoms and quality of life were assessed. Sociodemographic variables were also collected.

\section{Intervention}

The structured medication assessment included a review of medication, an assessment of the clinical information, and a meeting of an interprofessional team consisting of a pharmacist, physician and registered nurse working regularly in home care; they conducted the medication assessment within two weeks after the baseline measurements. Patients' updated and verified medication lists, baseline measurements, and electric medical records including their medical histories were available during the assessment.

Before the team meeting, the pharmacist reviewed the patients' medication lists using four databases: SFINX ${ }^{\circ}$ (currently INXBASE ${ }^{\circ}$ ) for drug-drug-interactions, PHAR $\mathrm{AO}^{\circ}$ (currently RISKBASE ${ }^{\circ}$ ) that complements SFINX ${ }^{\circ}$ with regard to 11 clinically relevant adverse effects, RENBASE $^{\circ}$ for renal risks [14] and the Database of Medication for the Elderly (Meds75+) [15]. The physician gathered information from medical records and on the patients' current clinical status.

The interprofessional team meeting discussed the patient's current health status and functioning and reviewed the patient's medications. The physician made clinical decisions and wrote conclusions into the patient's medical records at the end of the team meeting. The nurse updated the medication lists and informed patient about the changes; if necessary, the patient could participate in the interprofessional team meeting. The average time for the interprofessional team meeting was $20 \mathrm{~min}$, with the structured review done by the pharmacist lasting $27 \mathrm{~min}$. 


\section{BASELINE}

597 Eligible

83 Declined to participate

2 Did not meet inclusion criteria

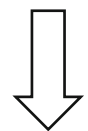

RANDOMIZATION

$n=512$

\begin{tabular}{|l|l|}
\hline INTERVENTION \\
$\begin{array}{l}\text { Interprofessional } \\
\text { Mediation } \\
\text { Assessment } \\
177 \text { women } \\
81 \text { Men }\end{array}$ \\
\hline
\end{tabular}

12 women died

8 men died

2 women withdrew

1 man withdrew

2 women not contacted

3 men not contacted

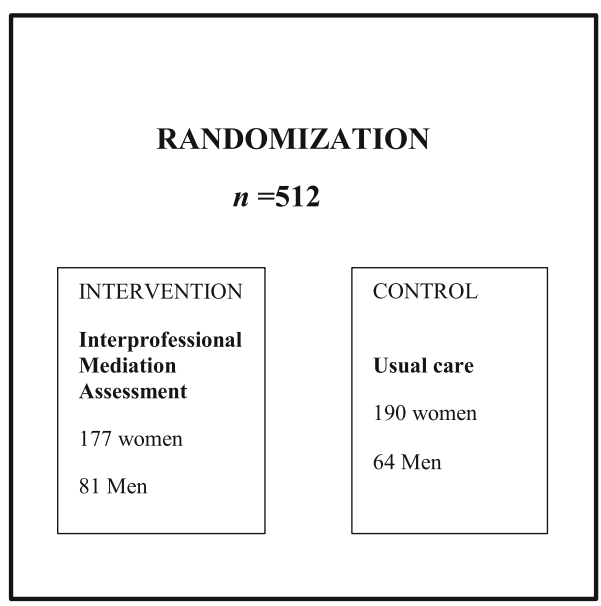

14 women died

9 men died

7 women withdrew

0 men withdrew

2 women not contacted

2 men not contacted

Fig. 1 Flow chart of the study

All pharmacists had a qualification in comprehensive medication review or current continuing professional development in clinical pharmacy. All interprofessional team members undertook a one-day training course or a personal introduction concerning the FIMA protocol.

\section{Usual care}

Patients randomised to usual care did not receive an interprofessional medication assessment. Information on their medication use was collected in a similar manner as in the intervention group but their baseline medication lists were 
reviewed by a pharmacist only after the six-month measurements had been conducted.

\section{Outcome measures}

Katz index of Activities of Daily Living (ADL) [16] and the Lawton and Brody scale of Instrumental Activities of Daily Living (IADL) scale [17] were used to evaluate each patient's performance. The maximum score in ADL is six; in IADL it is eight, with lower scores indicating increased requirement for assistance in daily activities. The Timed Up and Go (TUG) test was used to assess mobility, lower extremity strength and balance. The time taken to complete the TUG test correlates with the level of functional mobility [18]. The MiniMental State Examination (MMSE) was used for screening cognitive functions; MMSE scores $\leq 24$ indicate impaired cognitive function [19]. The Geriatric Depression Scale (GDS-15) was used for assessing depressive symptoms. Sum scores $\geq 6$ are suggestive of depression [20]. The preference-based, five-dimension instrument provided by EuroQol (EQ-5D-3L)(1) [21] was used for measuring health-related quality of life. These measurements were carried out at baseline and repeated at the six-month follow-up.

\section{Statistical methods}

Data were analysed according to randomisation group irrespective of whether the patients received the intervention as planned (the intention to treat principle). Baseline characteristics of the sample were summarized using proportions, percentages, and means with standard deviation (SD).

We used linear mixed models (LMM) with a random subject effect to detect differences in ADL, IADL, TUG, MMSE, GDS-15, and EQ-5D-3L between the usual care and intervention groups. Treatment (FIMA vs. usual care), time (baseline vs. 6-month follow-up), and sex served as factors, and age and CCI (excluding dementia) at baseline served as covariates. Based on our preliminary analyses (shown as request), the final models also included a treatmenttime-sex interaction. IBM $^{\circ}$ SPSS $^{\circ}$ Statistics Version 25 served as the statistical platform.

\section{Results}

\section{Participant characteristics}

The mean age of men was 83.1 (SD 6.9) and that of women was 85.2 (SD 6.2) years. Most participants, $64.1 \%$ of men and $81.2 \%$ of women, were living alone. The mean number of all medicines was 15 in both sexes, ranging from 2 to 36 in women and from 4 to 35 in men. The corresponding numbers for regularly consumed medicines were 9.5 (range: 1-20) in women and 9.6 (317) in men. At baseline, there were no statistically significant differences between intervention and usual care groups in either women or men (Table 1).

Table 1 Baseline characteristics of the study participants by sex and randomization status (intervention or usual care)

\begin{tabular}{|c|c|c|c|c|}
\hline & \multicolumn{2}{|l|}{ Women } & \multicolumn{2}{|l|}{ Men } \\
\hline & $\begin{array}{l}\text { Intervention } \\
n=177\end{array}$ & $\begin{array}{l}\text { Usual Care } \\
n=190\end{array}$ & $\begin{array}{l}\text { Intervention } \\
n=81\end{array}$ & $\begin{array}{l}\text { Usual Care } \\
n=64\end{array}$ \\
\hline Age (years), mean (SD) & $85.1(6.62)$ & $85.1(5.75)$ & $82.9(6.59)$ & $83.4(7.18)$ \\
\hline Living alone, $n(\%)$ & $149(84)$ & $149(78)$ & $53(65)$ & $40(63)$ \\
\hline \multicolumn{5}{|l|}{ Chronic diseases, $n$ (\%) } \\
\hline Cardiovascular diseases & $164(93)$ & $174(91)$ & $72(89)$ & $60(92)$ \\
\hline Diseases of musculoskeletal system & $115(65)$ & $125(65)$ & $43(53)$ & $30(46)$ \\
\hline Diabetes & $63(36)$ & $66(35)$ & $28(35)$ & $26(40)$ \\
\hline Cerebrovascular diseases & $48(27)$ & $54(28)$ & $35(43)$ & $32(49)$ \\
\hline Dementia & $62(35)$ & $62(33)$ & $23(28)$ & $14(22)$ \\
\hline Asthma or chronic obstructive pulmonary disease & $29(16)$ & $41(16)$ & $23(28)$ & $12(19)$ \\
\hline Psychiatric diseases & $34(19)$ & $32(17)$ & $15(19)$ & $7(11)$ \\
\hline Cancer & $24(14)$ & $21(11)$ & $22(28)$ & $12(19)$ \\
\hline Gastrointestinal diseases & $30(17)$ & $28(15)$ & $11(14)$ & $8(12)$ \\
\hline Neurological diseases & $23(13)$ & $20(11)$ & $13(16)$ & $12(19)$ \\
\hline Charlson Comorbidity Index, mean (SD) & $3.05(2.48)$ & $2.65(2.26)$ & $3.81(3.09)$ & $3.22(2.41)$ \\
\hline All medicines ${ }^{a}$, mean (SD) & $15(5.4)$ & $16(5.2)$ & $15(4.8)$ & $15(4.6)$ \\
\hline Regularly taken & $9(3.1)$ & $10(3.0)$ & $10(2.9)$ & $10(3.1)$ \\
\hline Taken as needed & $3(2.7)$ & $4(2.6)$ & $4(2.9)$ & $3(2.2)$ \\
\hline
\end{tabular}

\footnotetext{
${ }^{a}$ Including prescription and over-the-counter medicines
} 
Impact on functioning and health-related quality of life In the usual care group, the TUG time worsened on average by $2.4 \mathrm{~s}$ (i.e. the time increased) between baseline and follow-up measurements whereas in the FIMA group, the TUG time improved on average by $1.0 \mathrm{~s}$ (Table 2 and Fig. 2). With regard to ADL, the LMM detected a statistically significant time-treatment-sex interaction (Table 2). The ADL score decreased in both groups and both sexes, but the decline was steepest among women belonging to the FIMA group (Fig. 2).

\section{Discussion}

We investigated the impact of an interprofessional medication assessment on home care patients' functioning in a randomised, controlled study design. Based on the mean changes in LMMs, the FIMA intervention appeared to prevent worsening of TUG performance among older home care patients. During the six-month follow-up, functioning as assessed by ADL declined. Instrumental activities, cognitive functioning, mood and quality of life did not exhibit any significant changes during the follow-up. These findings are in line with the results of a systematic review and meta-analysis of randomised, controlled trials involving a medication review as an isolated short-term intervention [22].

In our study, changes in TUG times were different in the intervention and usual care groups favoring the intervention. In general, TUG performance is better among men than women [23] and our results were concordant with this fact. In previous studies, longer TUG times have been associated with lower executive function performance, risk of falls, functional declines and frailty [24-26]. A Canadian study [24] examining older, communitydwelling people, found that the longer it took to complete the TUG test, the greater was the individual's risk for experiencing a decline in activities of daily living. The threemonth risk for a decline in daily functioning rose from 5 to 9 -fold when TUG times increased from 20 to $29 \mathrm{~s}$ to $\geq 30 \mathrm{~s}$. In addition, TUG times $\geq 30 \mathrm{~s}$ represented nearly a 4 -fold risk for frailty compared with TUG times $\leq 10 \mathrm{~s}$. Thus, enhancing mobilty and balance perfomance may decrease these risks.

Medication assessments have been examined in several studies in varying settings among older people. A systematic review and meta-analysis of randomised, controlled trials concerning the effectiveness of medication reviews [22] revealed minimal effects on clinical outcomes and no impact on the quality of life. However, the meta-analysis suggested that a medication review may decrease the number of falls, which supports our findings on mobility and balance performance. A Cochrane review concerning pharmacist services for non-hospitalised patients [27] concluded that pharmacist services may slightly improve physical functioning, but physical functioning measured by SPF-36 questionnaire seemed to be enhanced only in diagnosisspecific trials in patients approximately 48-66 years old. For example, two randomised, controlled medication review trials among older patients with multiple conditions found no change in physical functioning [28, 29]. Regarding the present and published findings, it does seem to be difficult to improve physical functioning via an isolated intervention in older people carrying a high disease burden.

The general trend in ADL and IADL functioning is downwards among older people. The clinically relevant change in ADL is considered to be 0.5 or 1.0 point [30] and our intervention could not prevent this decline. ADL scores declined in both groups, and against expectations, even more in the intervention than in the control group. However, the changes in the mean ADL scores were less than 0.5 points.

Our study has several strengths. It was a randomised, controlled study in a real-life context. Furthermore, the study has a practice-based design with a public home care team conducting a pragmatic intervention. In addition, medication assessments are particularly indicated among home care patients. We assume that our findings are

Table 2 Functioning at baseline ( 0 month) and at the six-month follow-up (6 months) together with predicted change in functioning (LMM). In LMM with a random subject effect, treatment (FIMA vs. usual care), time (0 vs. 6 months), and sex served as factors, and age and $\mathrm{CCl}$ (excluding dementia) at baseline served as covariates. Values at 0 month and 6 months indicate crude mean \pm SD, predicted values indicate mean, $95 \% \mathrm{Cl}$. P-values refer to the treatment-time-sex interaction

\begin{tabular}{|c|c|c|c|c|c|c|c|}
\hline & \multicolumn{3}{|l|}{ FIMA } & \multicolumn{3}{|l|}{ Usual Care } & \multirow[t]{2}{*}{$\mathbf{P}$} \\
\hline & 0 month & 6 months & LMM & 0 month & 6 months & LMM & \\
\hline$\overline{A D L}$ & $4.98 \pm 1.30$ & $4.71 \pm 1.49$ & $-0.30,-0.32-(-0.28)$ & $4.82 \pm 1.27$ & $4.78 \pm 1.37$ & $-0.09,-0.09-(-0.09)$ & 0.026 \\
\hline IADL & $4.05 \pm 2.01$ & $3.73 \pm 2.11$ & $-0.34,-0.35-(-0.34)$ & $4.05 \pm 2.06$ & $4.00 \pm 2.14$ & $-0.12,-0.13-(-0.11)$ & 0.217 \\
\hline TUG, s & $28.6 \pm 28.2$ & $26.6 \pm 22.3$ & $-0.98,-1.03-(-0.93)$ & $25.5 \pm 15.9$ & $26.6 \pm 17.7$ & $2.35,2.24-2.47$ & 0.135 \\
\hline MMSE & $22.4 \pm 4.59$ & $22.2 \pm 4.84$ & $-0.39,-0.41-(-0.37)$ & $22.7 \pm 4.68$ & $22.5 \pm 4.64$ & $-0.24,-0.28-(-0.20)$ & 0.194 \\
\hline GDS-15 & $5.43 \pm 3.20$ & $5.27 \pm 3.18$ & $-0.10,-0.14-(-0.05)$ & $4.95 \pm 3.10$ & $5.00 \pm 3.03$ & $0.14,0.13-0.14$ & 0.121 \\
\hline EQ-5D-3L & $0.58 \pm 0.25$ & $0.57 \pm 0.29$ & $-0.017,-0.019-(-0.016)$ & $0.59 \pm 0.25$ & $0.56 \pm 0.27$ & $-0.023,-0.026-(-0.020)$ & 0.589 \\
\hline
\end{tabular}

LMM Linear Mixed Models, FIMA The Finnish Interprofessional Medication Assessment, ADL Katz index of Activities of Daily Living, IADL Lawton and Brody scale of Instrumental Activities of Daily Living, TUG Timed up and go test MMSE Mini-Mental State Examination, GDS-15 Geriatric depression scale, EQ-5D-3L Health-related quality of life 


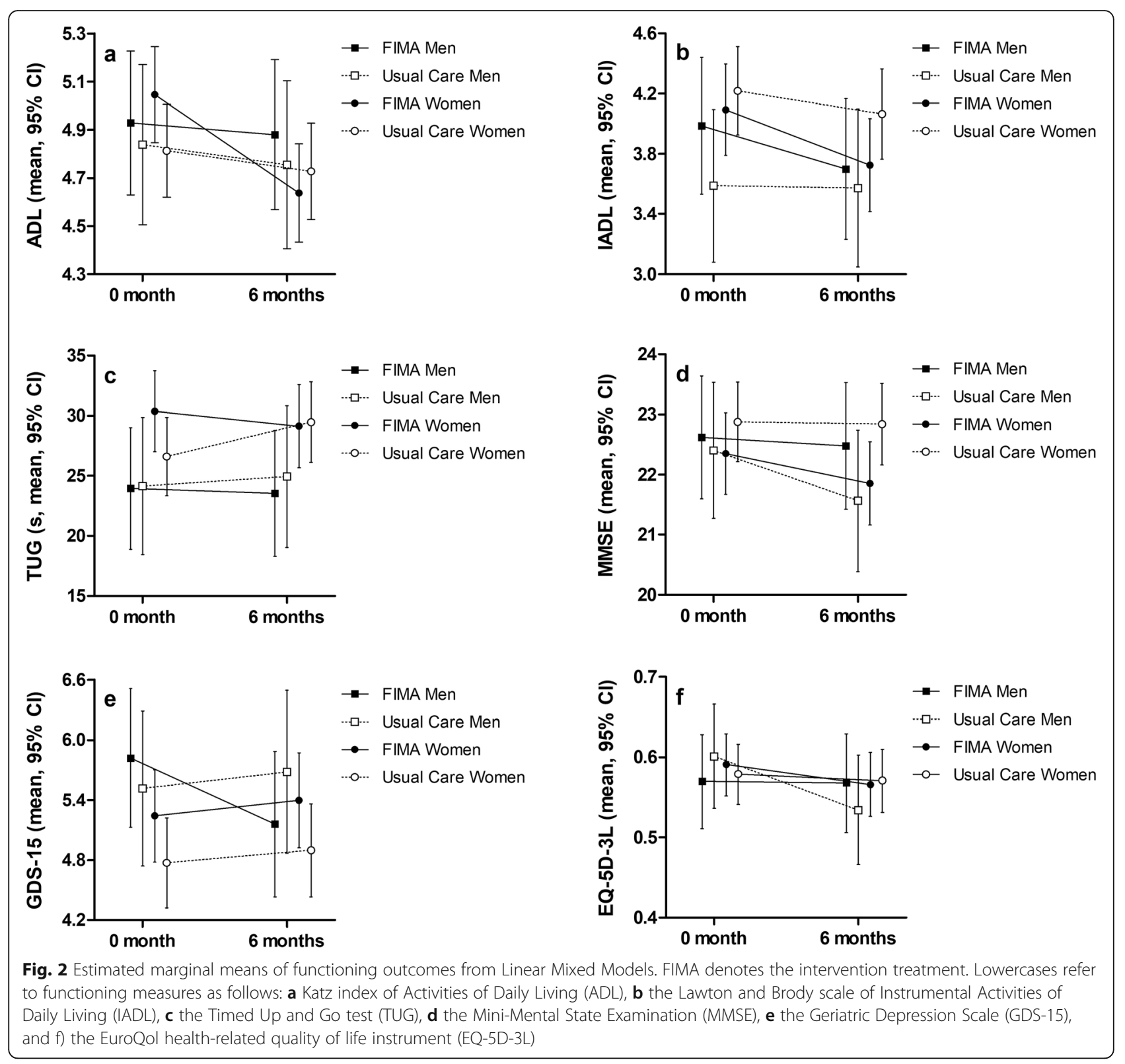

generalizable to older home care patients receiving polypharmacy and who are at an increased risk of falls. We used well-known, validated outcome measures to examine different dimensions of functioning. The detection and assessment of medication related risks and interactions were based on four decision support systems that are available and commonly used in the Finnish health care system.

The FIMA procedure has been extensively described and is transferable to different contexts. The procedure has been devised by public health care professionals, which means that information on patient's clinical conditions is relevantly considered in the medication assessment. Furthermore, the physician can make changes to the patient's medication at the interprofessional team meeting, when all of his/her information is available. In the FIMA procedure, the home care nurse conducts the verification of the current medication that patient uses at home, which enables medication assessments for large number of patients in routine care. In addition to the records in the patient files, the nurse receives instructions for further follow-up directly at the interprofessional team meeting, which reduces the risks of misinformation and misunderstandings.

This study has some limitations. Due to the high age and multimorbidity, this sample was medically unstable, factors which may diminish the impact of any intervention, especially that of single-domain interventions. We assessed the impact of the medication assessment performed only once, although regular and repeated 
assessments would be preferable for vulnerable home care patients with changing levels of health and functioning. Nonetheless, it is not recommended to make several changes in older peoples' medications concurrently. In addition, the same home care teams treated both intervention and usual care patients, which may have caused an observer effect. Although all the interprofessional teams had a one-day training, there might have been some variations in working practices between the teams in the five towns.

\section{Conclusions}

To conclude, it is challenging to improve functioning or prevent functional declines among vulnerable home care patients. Nonetheless, our findings indicate that an interprofessional medication assessment can prevent worsening of mobility of older people receiving home care services.

\section{Abbreviations}

FIMA: The Finnish Interprofessional Medication Assessment; ADL: Activities of Daily Living; IADL: Instrumental Activities of Daily Living; TUG: Timed Up \& Go Test; MMSE: Mini-Mental State Examination; GDS-15: Geriatric Depression Scale; CCl: Charlson Comorbidity Index; EQ-5D-3L: EuroQol five-dimension instrument for health-related quality of life; SD: Standard deviation

\section{Acknowledgements}

We appreciate the work done by the health care personnel for conducting this study in all five study centers i.e. Forssa, Haapajärvi, Lahti, Juva and Savonlinna. The authors want to thank the FIMA study group: Johanna Räisänen, Antti Mäntylä, Hannes Enlund, Anne Kumpusalo-Vauhkonen, Merja Merikoski and Timo Liukkonen. The authors would also like to thank the home care patients who participated in the FIMA study.

\section{Authors' contributions}

$K A, A V, J J$, EL and PM conceived, designed and implemented the study. KA, AV and JJ collected and analyzed the data. KA, AV, JJ, EL and PM drafted the manuscript. All authors read and approved the final manuscript.

\section{Funding}

The FIMA Study concept, design and acquisition of data were funded by the Ministry of Social Affairs and Health, Finland. The preparation of the present manuscript was supported by the South Savo Regional Fund of the Finnish Cultural Foundation, Uulo Arhio Foundation and Outpatient Care Research Foundation.

\section{Availability of data and materials}

The datasets generated and analysed during the current study are not publicly available due national regulations and agreements obtained to perform the study but are available from the corresponding author on reasonable request. The data required to reproduce the present findings cannot be shared at this time as the data also forms part of an ongoing study.

\section{Ethics approval and consent to participate}

The Research Ethics Committee of Northern Savo Hospital District and Kuopio University Hospital approved the FIMA study protocol on February 3, 2015. The FIMA study was registered with Clinical Trials.gov on March 20, 2015 (identifier: NCT02398812). Written informed consent was obtained from all individual patients included in the study or their closest proxy if the patient had a cognitive impairment.

\section{Consent for publication}

Not applicable.

\section{Competing interests}

The authors declare that they have no competing interests.

\section{Author details}

${ }^{1}$ The East Savo Hospital District, BOX 111, Fl-57101 Savonlinna, Finland. ${ }^{2}$ Institute of Public Health and Clinical Nutrition, Faculty of Health Sciences, University of Eastern Finland, Kuopio, Finland. ${ }^{3}$ Assessment of Pharmacotherapies, Finnish Medicines Agency, Kuopio, Finland. ${ }^{4}$ Kuopio University Hospital, Primary Heath Care Unit, Kuopio, Finland.

Received: 12 February 2020 Accepted: 28 September 2020

Published online: 06 October 2020

\section{References}

1. Maher RL, Hanlon J, Hajjar ER. Clinical consequences of polypharmacy in elderly. Expert Opin Drug Saf. 2014;13(1):57-65.

2. Landi F, Russo A, Liperoti R, Barillaro C, Danese P, Pahor M, Bernabei R, Onder G. Impact of inappropriate drug use on physical performance among a frail elderly population living in the community. Eur J Clin Pharmacol. 2007;63(8):791-9.

3. Zhang H, Du W, Gnjidic D, Chong S, Glasgow N. Trends in adverse drug reaction-related hospitalisations over 13 years in New South Wales. Australia Intern Med J. 2019:49(1):84-93.

4. Magaziner J, Cadigan DA, Fedder DO, Hebel JR. Medication use and functional decline among community-dwelling older women. J Aging Health. 1989;1(4):470-84

5. Spinewine A, Schmader KE, Barber N, Hughes $C$, Lapane $K L$, Swine $C$, Hanlon JT. Appropriate prescribing in elderly people: how well can it be measured and optimised? Lancet. 2007;370(9582):173-84.

6. Steinman MA, Hanlon JT. Managing medications in clinically complex elders: "There's got to be a happy medium". JAMA. 2010;304(14):1592-601.

7. Kaur S, Mitchell G, Vitetta L, Roberts MS. Interventions that can reduce inappropriate prescribing in the elderly: a systematic review. Drugs Aging. 2009;26(12):1013-28.

8. Topinkova E, Baeyens JP, Michel JP, Lang PO. Evidence-based strategies for the optimization of pharmacotherapy in older people. Drugs Aging. 2012; 29(6):477-94.

9. Patterson Susan M, Cadogan Cathal A, Kerse N, Cardwell Chris R, Bradley Marie C, Ryan C, Hughes C. Interventions to improve the appropriate use of polypharmacy for older people. Cochrane Database Syst Rew. 2014;(10).

10. Kallio S, Kumpusalo-Vauhkonen A, Jarvensivu T, Mantyla A, PohjanoksaMantyla M, Airaksinen M. Towards interprofessional networking in medication management of the aged: current challenges and potential solutions in Finland. Scand J Prim Health Care. 2016;34(4):368-76.

11. Auvinen K, Raisanen J, Merikoski M, Mantyla A, Kumpusalo-Vauhkonen A, Enlund H, Liukkonen T, Jyrkka J, Lonnroos E, Mantyselka P. The Finnish Interprofessional medication assessment (FIMA): baseline findings from home care setting. Aging Clin Exp Res. 2018;31(10):1471-9.

12. Auvinen $K$, Räisänen J, Voutilainen $A$, Jyrkkä J, Mäntyselkä $P$, Lönnroos $E$. Interprofessional medication assessment has effects on the quality of medication among home care patients: randomized controlled intervention study. J Am Med Dir Assoc. 2020, ISSN 1525-8610. https://doi.org/10.1016/j. jamda.2020.07.007.

13. Charlson ME, Pompei $P$, Ales $K L$, Mackenzie CR. A new method of classifying prognostic comorbidity in longitudinal studies: development and validation. J Chronic Dis. 1987:40(5):373-83.

14. Medbase. http://www.medbase.fi/en/professionals/. Accessed 31 Jan 2017

15. Meds75+. https://www.fimea.fi/web/en/databases_and_registeries/ medicines_information/database_of_medication_for_the_elderly. Accessed 31 Jan 2017.

16. Katz S. Akpom CA: 12. Index of ADL Med Care. 1976;14(5 Suppl):116-8.

17. Lawton MP, Brody EM. Assessment of older people: self-maintaining and instrumental activities of daily living. Gerontologist. 1969;9(3):179-86.

18. Shumway-Cook A, Brauer S, Woollacott M. Predicting the probability for falls in community-dwelling older adults using the timed up \& go test. Phys Ther. 2000;80(9):896-903.

19. Folstein MF, Folstein SE, McHugh PR. "mini-mental state". A practical method for grading the cognitive state of patients for the clinician. J Psychiatr Res. 1975;12(3):189-98.

20. Smarr KL, Keefer AL. Measures of depression and depressive symptoms: Beck depression inventory-II (BDI-II), Center for Epidemiologic Studies Depression Scale (CES-D), geriatric depression scale (GDS), hospital anxiety and depression scale (HADS), and patient health Questionnaire-9 (PHQ-9). Arthritis Care Res (Hoboken). 2011;63(Suppl 11):S454-66. 
21. Rabin R, de Charro F. EQ-5D: a measure of health status from the EuroQol group. Ann Med. 2001;33(5):337-43.

22. Huiskes VJ, Burger DM, van den Ende CH, van den Bemt BJ. Effectiveness of medication review: a systematic review and meta-analysis of randomized controlled trials. BMC Fam Pract. 2017;18(1):5 016-0577-x.

23. Ibrahim A, Singh DKA, Shahar S. 'Timed Up and Go' test: Age, gender and cognitive impairment stratified normative values of older adults. PLoS One. 2017;12(10):e0185641. Published 2017 Oct 3. https://doi.org/10.1371/journal. pone.0185641

24. Eagles D, Perry JJ, Sirois M, Lang E, Daoust R, Lee J, Griffith L, Wilding L, Neveu X, Emond M. Timed up and go predicts functional decline in older patients presenting to the emergency department following minor trauma. Age Ageing. 2016:46(2):214-8.

25. Boye ND, Mattace-Raso FU, Van Lieshout EM, Hartholt KA, Van Beeck EF, Van der Cammen TJ. Physical performance and quality of life in single and recurrent fallers: data from the improving medication prescribing to reduce risk of falls study. Geriatr Gerontol Int. 2015;15(3):350-5.

26. McGough EL, Kelly VE, Logsdon RG, McCurry SM, Cochrane BB, Engel JM, Teri L. Associations between physical performance and executive function in older adults with mild cognitive impairment: gait speed and the timed "up \& go" test. Phys Ther. 2011;91(8):1198-207.

27. de Barra M, Scott CL, Scott NW, Johnston M, de Bruin M, Nkansah N, Bond CM, Matheson Cl, Rackow P, Williams AJ, Watson MC. Pharmacist services for nonhospitalised patients. Cochrane Database Syst Rev. 2018:9:CD013102.

28. Sellors J, Kaczorowski J, Sellors C, Dolovich L, Woodward C, Willan A, Goeree R, Cosby R, Trim K, Sebaldt R, Howard M, Hardcastle L, Poston J. A randomized controlled trial of a pharmacist consultation program for family physicians and their elderly patients. CMAJ. 2003;169(1):17-22.

29. Williams ME, Pulliam CC, Hunter R, Johnson TM, Owens JE, Kincaid J, Porter C, Koch G. The short-term effect of interdisciplinary medication review on function and cost in ambulatory elderly people. J Am Geriatr Soc. 2004; 52(1):93-8.

30. Suijker JJ, van Rijn M, Ter Riet G, Moll van Charante EP, de Rooij SE, Buurman BM. Minimal important change and minimal detectable change in activities of daily living in community-living older people. J Nutr Health Aging. 2017;21(2):165-72

\section{Publisher's Note}

Springer Nature remains neutral with regard to jurisdictional claims in published maps and institutional affiliations.

Ready to submit your research? Choose BMC and benefit from:

- fast, convenient online submission

- thorough peer review by experienced researchers in your field

- rapid publication on acceptance

- support for research data, including large and complex data types

- gold Open Access which fosters wider collaboration and increased citations

- maximum visibility for your research: over $100 \mathrm{M}$ website views per year

At $\mathrm{BMC}$, research is always in progress.

Learn more biomedcentral.com/submissions 